\title{
Journal of Surgery
}

Chen WS, et al. J Surg: JSUR-1179.

\section{Treatment of Esophagogastric Anastomotic Leak and Bronchomedi- astinal Esophageal Fistula with a Deltopectoral Flap and Hyperbaric Oxygen Therapy: A Case Report}

\author{
Wei-Shan Chen, 1 Huang-Kai Kao2, Kun-Ju Yang, $3^{*}$, Yun-Hen Liu1
}

1Department of Thoracic Surgery, Chang Gung Memorial Hospital, Chang Gung University, Tao-Yuan, Taiwan

${ }_{2}$ Department of Plastic and Reconstructive Surgery, Chang Gung Memorial Hospital, Chang Gung University, Tao-Yuan, Taiwan

${ }_{3}$ Division of Hyperbaric Oxygen Center, Department of Emergency Medicine, Chang Gung Memorial Hospital, Chang Gung

University, Tao-Yuan, Taiwan

*Corresponding author: Kun-Ju Yang, Division of Hyperbaric Oxygen Center, Department of Emergent Medicine, Chang Gung Memorial Hospital and Chang Gung University, 5 Fuxing St., Guishan Dist., Taoyuan City, 333, Taiwan. Tel: +88633281200 Ext: 2505; Email: kunju@adm.cgmh.org.tw

Citation: Chen WS, Kao HK, Yang KJ, Liu YH (2018) Treatment of Esophagogastric Anastomotic Leak and Bronchomediastinal Esophageal Fistula with a Deltopectoral Flap and Hyperbaric Oxygen Therapy: A Case Report. J Surg: JSUR-1179. DOI: $10.29011 / 2575-9760.001179$

Received Date: 15 September, 2018; Accepted Date: 19 September, 2018; Published Date: 25 September, 2018

\begin{abstract}
Esophagogastric anastomotic leaks with inflammatory involvement of the trachea-bronchial tree is one of the most difficult complication after esophagectomy. We report a case of anastomotic leak with a huge fistula connecting the esophagus, posterior mediastinum, and left main bronchus. The condition was successfully treated with a deltopectoral flap, T-tube drainage, and hy-perbaric oxygen therapy. Based on our preliminary experience, we believed that HBO therapy could be considered to be included in the treatment of tracheo-esophageal fistula, especially when the lesion is large or complicated and difficult to treat completely with conventional interventions.
\end{abstract}

Keywords: Anastomotic Leak; Fistula; Hyperbaric Oxygenation; Surgical Flaps; Esophagectomy

\section{Introduction}

Esophagogastric anastomotic leaks after esophagectomy are potentially life threatening. Various complications have been reported, and the most difficult one is leakage from the esophagogastric anastomosis with inflammatory involvement of the trachea-bronchial tree [1]. We report a case of anastomotic leak with a huge fistula connecting the esophagus, posterior mediastinum, and left main bronchus. The condition was successfully treated with a deltopectoral flap, T-tube drainage, and Hyperbaric Oxygen (HBO) therapy.

\section{Case Presentation}

A 61-year-old man with esophageal cancer underwent esophagectomy and gastric tube reconstruction, and his postoperative recovery was smooth at first. However, he presented with high fever, dyspnea, and persisting productive cough one month after the surgery. He was admitted for suspicion of pneumonia, which was later found to be induced by a bronchomediastinal esophageal fistula. Bronchoscopy revealed one fistula opening located at the left main bronchus, $1 \mathrm{~cm}$ beneath the carina. Panendoscopy showed a blind loop developing from the site of esophagogastric anastomosis. The fistula opening was found in the blind loop at $25 \mathrm{~cm}$ beneath the incisor. Computed Tomography (CT) of the thorax revealed the dimensions of the whole fistula: a $5 \mathrm{~mm}$ opening between the esophagus and mediastinum, $2 \mathrm{~cm}$ in maximum width, $11 \mathrm{~cm}$ in total length, and a $5 \mathrm{~mm}$ opening between the mediastinum and left main bronchus. We performed a treatment combining thoracic surgery, plastic surgery, and HBO therapy. Under the guidance of endoscope, a nasogastric tube was first inserted as a guide into the esophagus. We then approached through the previous surgery wound on the left side of the neck. The esophagogastric anastomotic leak was found above the clavicle level. A T-tube was then inserted into the leakage wound. Afterwards, a plastic surgeon harvested a 5-cm-wide

J Surg, an open access journal 
left deltopectoral flap and obliterated the esophageal-mediastinal fistula opening (Figure 1).

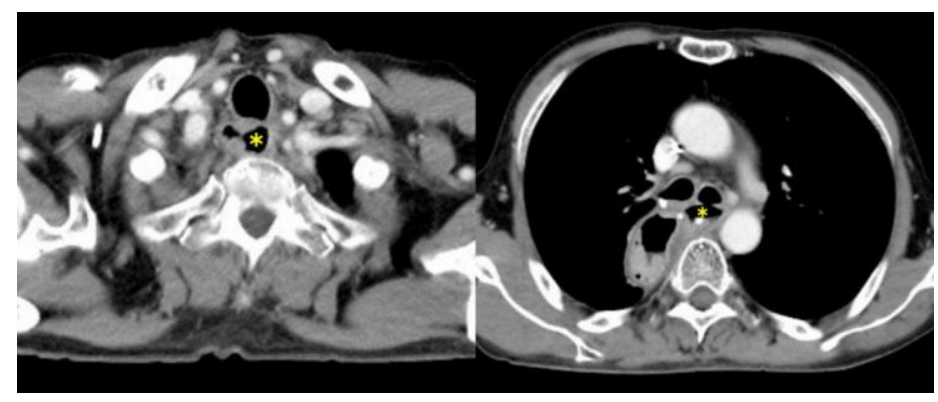

Figure 1: CT showing the fistula (asterisk) with esophageal-mediastinal and mediastinal-bronchial connection.

The whole surgery ended with no air leakage from the repair site and the T-tube draining out through the surgery wound. One week after the surgery, the patient started to receive HBO therapy. A total of 20 treatments were applied for 1 month once every day and 5 days per week. Every treatment was performed at a pressure of 2.0 atmospheres for 80 minutes in a monoplace hyperbaric chamber. One month after finishing HBO therapy, we removed the T-tube. Post-treatment CT revealed marked regression, and the previous fistula was no longer observed at the same position. Panendoscopy showed good healing of the fistula with the muscle flap over the previous anastomotic leak (Figures 2,3). Bronchoscopy also revealed complete healing of the fistula. At the latest follow-up (1-year post-operative), the patient showed satisfactory oral intake status without respiratory symptoms or recurrence of other fistulas.

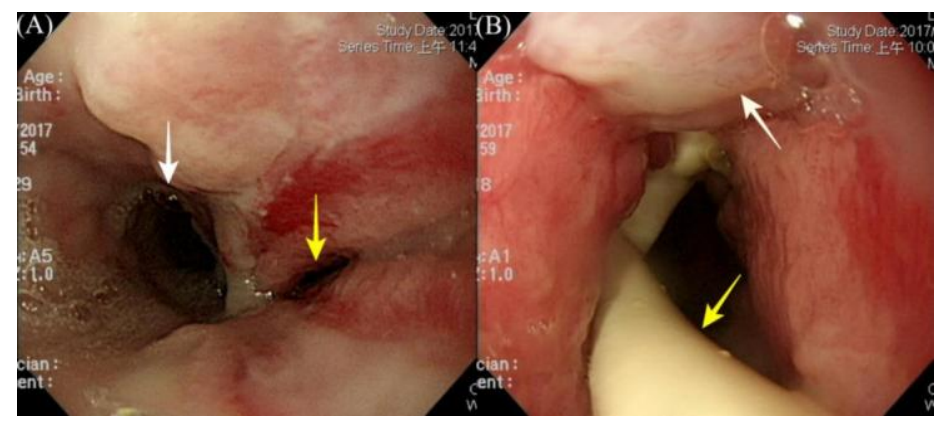

Figure 2: Panendoscopy showing the esophageal-mediastinal fistula. (A) Pre-operation. White arrow: esophageal-mediastinal fistula. Yellow arrow: anastomotic leak region. (B) Post-operation. White arrow: the delto-pectoralis muscle flap covering the anastomotic leak region. Yellow arrow: the T-tube inserted in the previous anastomotic leak site to drain out saliva.

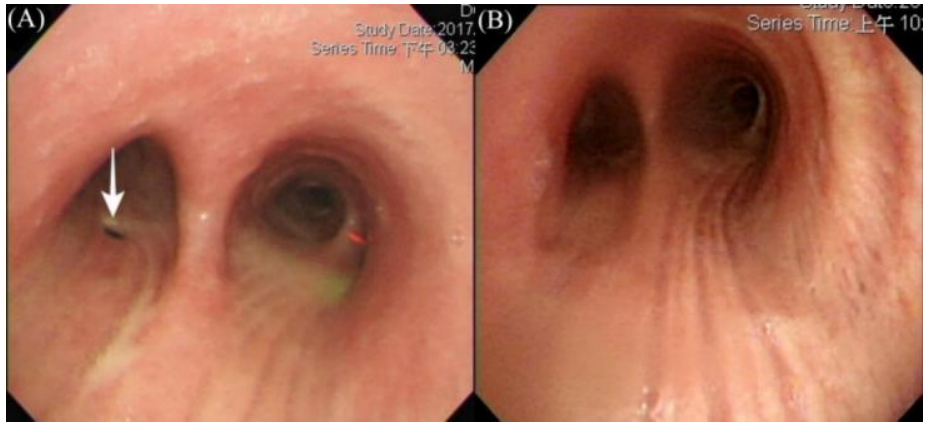

Figure 3: Bronchoscopy showing the mediastinal-bronchial fistula opening. (A) Pre-operation. White arrow: mediastinal-bronchial fistula opening at the left main bronchus, $1 \mathrm{~cm}$ beneath the carina. (B) Postoperation. No endobronchial lesion at the previous site.

\section{Discussion}

The treatment of a tracheo-esophageal fistula following anastomotic leakage after esophagectomy depends on the site and size of the fistula and the severity of the symptoms. Patients without signs of infection may receive conservative treatment like oral antimicrobial agents. As for severe cases, surgical intervention is necessary. The most common surgical procedure is the direct repair of tracheal and esophageal defects with the interposition of a pedicled soft tissue flap to prevent recurrent fistulization. If the gastric tube cannot be preserved due to necrosis, it may be excised and reconstructed with colonic interposition [1]. Endoscopic sealing of fistula using covered expandable metallic stents has also shown encouraging results [2]. In our case, the bulky size of the whole fistula made direct repair and interposition of the flap difficult. For the esophageal-mediastinum fistula, we did not consider placing metallic stents to avoid pain and the sensation of a foreign body, because the leakage region was located at the upper esophageal sphincter. Diversion of the esophagus with later colonic reconstruction was not suitable either because of its high position. Therefore, we used the T-tube to drain out the saliva and covered the esophageal-mediastinum fistula opening with a muscle flap, thereby avoiding persistent irritation by the saliva.

With regard to the broncho-mediastinal fistula, treatments include stent insertion and the application of tissue glues. Nonetheless, the risk of these treatments is blockage of the mediastinal abscess drainages via the bronchial fistula wound, which would lead to severe infection and mediastinitis. HBO therapy enhances epithelization, neovascularization, collagen deposition and wound healing [3]. It has been successfully used in the management of esophagotracheal fistula caused by lithium 
Citation: Chen WS, Kao HK, Yang KJ, Liu YH (2018) Treatment of Esophagogastric Anastomotic Leak and Bronchomediastinal Esophageal Fistula with a Deltopectoral Flap and Hyperbaric Oxygen Therapy: A Case Report. J Surg: JSUR-1179. DOI: 10.29011/2575-9760.001179

disc battery ingestion [3]. Hence, we utilized HBO therapy to treat the mediastinal-bronchial fistula. In conclusion, our case was a successful experience of combining muscle flap interposition and HBO therapy to treat a huge fistula with esophageal-mediastinal and mediastinal-bronchial connection. Based on our preliminary experience, we believe that HBO therapy could be considered to be included in the treatment of tracheo-esophageal fistula, especially when the lesion is large or complicated and difficult to treat completely with conventional interventions.

\section{References}

1. Buskens JC, Hulscher JBF, Fockens $P$, Obertop H, van Lanschot JJ (2001) Benign tracheo-neo-esophageal fistulas after subtotal esophagectomy. Ann Thorac Surg 72: 221-224.

2. Hoon JH, Song HY, Ko GY, Lim JO, Yoon HK, et al. (2004) Esophagorespiratory fiistula; long-term results of palliative treatment with covered expandable metallic stents in 61 patients. Radiology 232: 252-259.

3. Petri NM, Mestrović J, Andrić D, Krzelj V, Stipancević H (2003) Esophagotracheal fistula after lithium disc battery ingestion successfully treated with hyperbaric oxygen therapy. Int J Pediatr Otorhinolaryngol 67: 921-926. 